\title{
PALETTE REORDERING UNDER AN EXPONENTIAL POWER DISTRIBUTION MODEL OF PREDICTION RESIDUALS
}

\author{
Armando J. Pinho and António J. R. Neves \\ Dept. de Electrónica e Telecomunicações / IEETA \\ Universidade de Aveiro, 3810-193 Aveiro, Portugal \\ ap@det.ua.pt - andieeta.pt
}

\begin{abstract}
Palette reordering is one of the most effective approaches for improving the compression of color-indexed images. Recently, a theoretically motivated modification of a reordering technique proposed by Zeng et al. was suggested, based on an exponential distribution model of the prediction residuals. In this paper, we develop this theoretical analysis further, exploiting a broader model based on exponential power distributions.
\end{abstract}

\section{INTRODUCTION}

The compression of color-indexed images is very demanding for most general purpose continuous-tone image coding techniques. Specialized approaches for coding colorindexed images do exist (see, for example, [1, 2, 3, 4]). However, it remains an important topic to ensure that general purpose image coding techniques, such as JPEG-LS $[5,6]$ or lossless JPEG $2000[7,8]$, do produce acceptable results with this class of images.

Color-indexed images are represented by a matrix of indexes (the index image) and by a color-map or palette. The indexes in the matrix address positions in the color-map and, therefore, establish the colors of the corresponding pixels. For a particular image, the mapping between index values and colors is not unique. In fact, it can be arbitrarily permuted, under the condition that the corresponding index image is changed accordingly.

Palette reordering is a class of preprocessing methods, having the goal of finding a permutation of the color palette such that the resulting image of indexes is more suitable for compression. If the optimal configuration is sought, then the computational complexity involved can be high. In fact, the number of possible configurations for a table of $M$ colors corresponds to the number of permutations of $M$ objects, which equals $M !$. Therefore, exhaustive search is impractical for most of the interesting cases, which motivated sev-

This work was supported in part by the Fundação para a Ciência e a Tecnologia (FCT). eral sub-optimal, lower complexity, proposals $[9,10,11,12$, $13,14,15,16,17]$.

Recently, a theoretically motivated modification of a reordering method proposed by Zeng et al. [14] was suggested [17]. By assuming that the first order prediction residuals are frequently well modeled by an exponential distribution, a new set of parameters was proposed, leading to important improvements in the lossless compression of the index images [17]. In this paper, we develop the theory further, by broadening the underlying distribution model and by studying, in practice, how appropriate is the exponential distribution that was assumed in [17]. This is done through the adoption of an exponential power distribution model which, as a particular case, includes the exponential distribution.

\section{ZENG'S METHOD}

The palette re-indexing method proposed by Zeng et al. [14] is based on an one-step look-ahead greedy approach, which aims at increasing the lossless compression efficiency of color-indexed images.

The algorithm starts by finding the index that is most frequently located adjacent to other (different) indexes, and the index that is most frequently found adjacent to it. This pair of indexes is the starting base for an ordered set, $\mathcal{S}$, that will be constructed, one index at a time, during the operation of the re-indexing algorithm. We denote by $v_{i}$ the indexes already assigned to the ordered set ( $i$ indicates the position of the index in the ordered set and, therefore, its distance to the left end side of the set) and by $u$ those still unassigned. Therefore, just before starting the iterations, $\mathcal{S}=\left\{v_{1}, v_{2}\right\}$, where $v_{1}$ and $v_{2}$ are the two indexes mentioned above. New indexes can only be attached to the left or to the right extremity of the ordered set.

The algorithm then proceeds as follows. For each iteration, compute $u_{L}$ and $u_{R}$ according to:

$$
u_{L}=\arg \max _{u \notin \mathcal{S}} D_{L}(u),
$$


where

$$
D_{L}(u)=\sum_{v_{i} \in \mathcal{S}} \alpha_{i} C\left(u, v_{i}\right)
$$

and

$$
u_{R}=\arg \max _{u \notin \mathcal{S}} D_{R}(u)
$$

where

$$
D_{R}(u)=\sum_{v_{i} \in \mathcal{S}} \alpha_{|\mathcal{S}|-i+1} C\left(u, v_{i}\right) .
$$

The function $C(i, j)=C(j, i)$ denotes the number of occurrences (measured in the initial index image) corresponding to pixels with index $i$ that are spatially adjacent to pixels with index $j$. The $\alpha_{k}$ are weights controlling the impact of the $C\left(u, v_{k}\right)$ on $D_{L}(u)$ and $D_{R}(u)$ and, originally [14], were proposed to be given by

$$
\alpha_{k}=\log _{2}\left(1+\frac{1}{k}\right)
$$

The new set is given by $\left\{u_{L}, v_{1}, \ldots, v_{|S|}\right\}$, if $D_{L}\left(u_{L}\right)>$ $D_{R}\left(u_{R}\right)$, or by $\left\{v_{1}, \ldots, v_{|S|}, u_{R}\right\}$, otherwise. This iterative process continues until assigning all indexes. Finally, the re-indexed image is constructed by applying the mapping $v_{i} \mapsto(i-1)$ to all image pixels, and changing the color-map accordingly.

\section{GENERALIZED ZENG'S METHOD}

In [17], a modification of Zeng's algorithm was proposed, relying on an exponential model for the distribution of first order prediction residuals, and on the assumption that the entropy of the absolute differences between neighboring pixels is a good indicator of the degree of compressibility of an image. In what follows, we extend the work reported in [17] in order to accommodate an exponential power distribution model of the prediction residuals.

According to the greedy strategy of Zeng's algorithm, the next index, $\bar{u}$, that should integrate $\mathcal{S}$ is the one that implies the largest increase in code length if its choice is postponed to the next iteration. It is well-known that, for a memoryless source, the number of bits required to represent the occurrence of a given symbol $s$ is given by $-\log _{2} P(s)$, where $P(s)$ denotes the probability of occurrence of $s$.

We start by defining the estimated code length implied by placing index $u$ on the left end side of $\mathcal{S}$

$$
l_{L}(u)=-\sum_{v_{i} \in \mathcal{S}} C\left(u, v_{i}\right) \log _{2} P(i),
$$

by placing it one position farther away

$$
l_{L}^{+}(u)=-\sum_{v_{i} \in \mathcal{S}} C\left(u, v_{i}\right) \log _{2} P(i+1),
$$

by placing it on the right end side of $\mathcal{S}$

$$
l_{R}(u)=-\sum_{v_{i} \in \mathcal{S}} C\left(u, v_{i}\right) \log _{2} P(|\mathcal{S}|-i+1),
$$

and by placing it one position farther away from the right end side

$$
l_{R}^{+}(u)=-\sum_{v_{i} \in \mathcal{S}} C\left(u, v_{i}\right) \log _{2} P(|\mathcal{S}|-i+2) .
$$

The new index, $\bar{u}$, should satisfy

$$
\bar{u}=\arg \max _{u \notin \mathcal{S}} \Delta l(u)
$$

with

$$
\Delta l(u)= \begin{cases}l_{L}^{+}(u)-l_{L}(u), & \text { if } l_{R}(u)-l_{L}(u)>0 \\ l_{R}^{+}(u)-l_{R}(u), & \text { otherwise. }\end{cases}
$$

In words, for each candidate index, $u$, its best position (left or right) is chosen, i.e., the one that minimizes the code length. Then, among all those indexes, we pick the one producing the largest increase in code length if its choice is postponed to the next iteration.

Now, we can write

$l_{L}^{+}(u)-l_{L}(u)=\sum_{v_{i} \in \mathcal{S}} \log _{2} \frac{P(i)}{P(i+1)} C\left(u, v_{i}\right)=\sum_{v_{i} \in \mathcal{S}} \alpha_{i} C\left(u, v_{i}\right)$,

if the best position for index $u$ is the left end side, or

$$
\begin{aligned}
l_{R}^{+}(u)-l_{R}(u) & =\sum_{v_{i} \in \mathcal{S}} \log _{2} \frac{P(|S|-i+1)}{P(|S|-i+2)} C\left(u, v_{i}\right)= \\
& =\sum_{v_{i} \in \mathcal{S}} \alpha_{|\mathcal{S}|-i+1} C\left(u, v_{i}\right),
\end{aligned}
$$

if the best position is the right end side, where

$$
\alpha_{k}=\log _{2} \frac{P(k)}{P(k+1)},
$$

and where $P(k)$ denotes the probability of occurrence of a difference of $k$ units between two neighboring pixels.

Moreover, we can also write

$$
\begin{aligned}
l_{R}(u)-l_{L}(u) & =\sum_{v_{i} \in \mathcal{S}}\left(\log _{2} P(i)-\log _{2} P(|\mathcal{S}|-i+1)\right) C\left(u, v_{i}\right)= \\
& =\sum_{v_{i} \in \mathcal{S}} \beta_{i} C\left(u, v_{i}\right),
\end{aligned}
$$

where

$$
\beta_{k}=\log _{2} \frac{P(k)}{P(|S|-k+1)} .
$$

For exponentially power distributed residuals, i.e., considering

$$
P(k)=A \theta^{k^{\gamma}}, \quad 0<\theta<1, \quad 0 \leq k<M, \quad \gamma>0
$$


Eq. (13) reduces to

$$
\alpha_{k}=\log _{2} \frac{A \theta^{k^{\gamma}}}{A \theta^{(k+1)^{\gamma}}}=\left(k^{\gamma}-(k+1)^{\gamma}\right) \log _{2} \theta
$$

and (15) to

$$
\beta_{k}=\log _{2} \frac{A \theta^{k^{\gamma}}}{A \theta^{(|S|-k+1)^{\gamma}}}=\left(k^{\gamma}-(|\mathcal{S}|-k+1)^{\gamma}\right) \log _{2} \theta
$$

Finally, we note that the $\log _{2} \theta$ term can be eliminated, since it is a constant factor, although bearing in mind that it is always negative.

\section{EXPERIMENTAL RESULTS AND DISCUSSION}

In this Section, we provide experimental results showing the compression gain that can be obtained if an exponential power model is used, in comparison to the exponential model (i.e., for $\gamma=1.0$ ). We give compression results for the same collection of color-indexed images that have been used in [17]. These are images both from synthetic and natural origins and of various sizes and number of colors. We provide results not only for a JPEG-LS encoder, as in [17], but also for a JPEG 2000 lossless encoder. Table 1 presents the compression results that have been obtained (both in terms of number of bytes and bits per pixel), including the size of the color-maps. The best value of $\gamma$ was determined for each image / encoder pair, and the compression gain in relation to the exponential distribution $(\gamma=1.0)$ is presented.

From the results presented in Table 1, we observe that, in fact, the exponential model seems to be a reasonable choice for most of the images. From the 30 test images included in Table 1, 17 (18 for JPEG 2000) of them had compression improvements of less than one percent. However, for some of the images ( 6 for JPEG-LS and 8 for JPEG 2000) the lossless compression gain was over $3 \%$.

A somewhat surprising observation is that, for $17 \mathrm{im}-$ ages, the best value of $\gamma$ is the same for both encoders, and for 6 others they differ only by 0.1 . This means that, apparently, for most of the images, the same distribution model is well suited for encoding engines so different as those of JPEG-LS (prediction-based) and JPEG 2000 (transformbased). In our opinion, this observation deserves further study, because it may contribute for establishing links between these two coding principles.

Finally, although currently it is not very practical to use the exponential power model for palette reordering (due to the need of searching for the best $\gamma$ for each image), it may be so if a low complexity way of guessing it from the image is found. This is a possibility that we plan to exploit in a near future.

\section{REFERENCES}

[1] P. J. Ausbeck Jr., "The piecewise-constant image model," Proceedings of the IEEE, vol. 88, no. 11, pp. 1779-1789, Nov. 2000.

[2] Y. Yoo, Y. G. Kwon, and A. Ortega, "Embedded image-domain compression using context models," in Proc. of the 6th IEEE Int. Conf. on Image Processing, ICIP-99, vol. I, Kobe, Japan, Oct. 1999, pp. 477-481.

[3] V. Ratnakar, "RAPP: Lossless image compression with runs of adaptive pixel patterns," in Proc. of the 32nd Asilomar Conf. on Signals, Systems, and Computers, 1998, vol. 2, 1998, pp. 1251-1255.

[4] X. Chen, S. Kwong, and J.-F. Feng, "A new compression scheme for color-quantized images," IEEE Trans. on Circuits and Systems for Video Technology, vol. 12, no. 10, pp. 904-908, Oct. 2002.

[5] Information technology - Lossless and near-lossless compression of continuous-tone still images, ISO/IEC 14495-1 and ITU Recommendation T.87, 1999.

[6] M. J. Weinberger, G. Seroussi, and G. Sapiro, "The LOCO-I lossless image compression algorithm: principles and standardization into JPEG-LS," IEEE Trans. on Image Processing, vol. 9, no. 8, pp. 13091324, Aug. 2000.

[7] Information technology - JPEG 2000 image coding system, ISO/IEC International Standard 15444-1, ITU-T Recommendation T.800, 2000.

[8] A. Skodras, C. Christopoulos, and T. Ebrahimi, "The JPEG 2000 still image compression standard," IEEE Signal Processing Magazine, vol. 18, no. 5, pp. 3658, Sept. 2001.

[9] A. Zaccarin and B. Liu, "A novel approach for coding color quantized images," IEEE Trans. on Image Processing, vol. 2, no. 4, pp. 442-453, Oct. 1993.

[10] L. M. Po and W. T. Tan, "Block address predictive colour quantisation image compression," Electronics Letters, vol. 30, no. 2, pp. 120-121, Jan. 1994.

[11] A. C. Hadenfeldt and K. Sayood, "Compression of color-mapped images," IEEE Trans. on Geoscience and Remote Sensing, vol. 32, no. 3, pp. 534-541, May 1994.

[12] P. Waldemar and T. A. Ramstad, "Subband coding of color images with limited palette size," in Proc. of the IEEE Int. Conf. on Acoustics, Speech, and Signal Processing, ICASSP-94, vol. V, Adelaide, Australia, Apr. 1994, pp. 353-356.

[13] N. D. Memon and A. Venkateswaran, "On ordering color maps for lossless predictive coding," IEEE Trans. on Image Processing, vol. 5, no. 5, pp. 15221527, Nov. 1996.

[14] W. Zeng, J. Li, and S. Lei, "An efficient color reindexing scheme for palette-based compression," in 


\begin{tabular}{|c|c|c|c|c|c|c|c|c|c|c|c|c|c|}
\hline \multirow{3}{*}{ Image } & \multirow{3}{*}{ Colors } & \multicolumn{6}{|c|}{ JPEG-LS } & \multicolumn{6}{|c|}{ JPEG 2000} \\
\hline & & \multicolumn{2}{|c|}{ mZeng $(\gamma=1.0)$} & \multicolumn{3}{|c|}{ Proposed } & \multirow{2}{*}{$\begin{array}{c}\text { Gain } \\
\%\end{array}$} & \multicolumn{2}{|c|}{ mZeng $(\gamma=1.0)$} & \multicolumn{3}{|c|}{ Proposed } & \multirow{2}{*}{$\begin{array}{c}\text { Gain } \\
\%\end{array}$} \\
\hline & & Size & bpp & Size & bpp & $\gamma$ & & Size & bpp & Size & bpp & $\gamma$ & \\
\hline $\mathrm{pc}$ & 6 & 320,479 & 0.745 & 320,479 & 0.745 & 1.0 & 0.0 & 322,560 & 0.749 & 322,560 & 0.749 & 1.0 & 0.0 \\
\hline books & 7 & 10,458 & 1.469 & 10,345 & 1.453 & 1.3 & 1.1 & 11,392 & 1.601 & 11,392 & 1.601 & 1.0 & 0.0 \\
\hline music & 8 & 1,620 & 1.051 & 1,620 & 1.051 & 1.0 & 0.0 & 2,117 & 1.374 & 2,117 & 1.374 & 1.0 & 0.0 \\
\hline winaw & 10 & 16,569 & 0.450 & 16,569 & 0.450 & 1.0 & 0.0 & 22,505 & 0.611 & 22,505 & 0.611 & 1.0 & 0.0 \\
\hline party8 & 12 & 5,993 & 0.318 & 5,959 & 0.316 & 2.3 & 0.6 & 7,782 & 0.413 & 7,626 & 0.405 & 2.3 & 2.0 \\
\hline netscape & 32 & 13,405 & 1.752 & 13,322 & 1.741 & 0.8 & 0.6 & 18,285 & 2.390 & 18,215 & 2.381 & 0.1 & 0.4 \\
\hline sea_dusk & 46 & 3,732 & 0.189 & 3,732 & 0.189 & 1.0 & 0.0 & 2,700 & 0.137 & 2,604 & 0.132 & 0.4 & 3.6 \\
\hline benjerry & 48 & 3,977 & 1.137 & 3,974 & 1.137 & 1.1 & 0.1 & 6,186 & 1.769 & 6,145 & 1.758 & 0.2 & 0.7 \\
\hline gate & 84 & 19,543 & 2.566 & 19,489 & 2.559 & 0.9 & 0.3 & 23,124 & 3.037 & 23,119 & 3.036 & 0.9 & 0.0 \\
\hline descent & 122 & 22,834 & 2.854 & 22,745 & 2.843 & 0.7 & 0.4 & 27,395 & 3.424 & 27,188 & 3.398 & 0.7 & 0.8 \\
\hline sunset & 204 & 88,610 & 2.307 & 88,126 & 2.294 & 1.2 & 0.6 & 125,416 & 3.266 & 124,929 & 3.253 & 1.2 & 0.4 \\
\hline yahoo & 229 & 6,072 & 1.789 & 5,995 & 1.767 & 0.5 & 1.3 & 7,646 & 2.253 & 7,513 & 2.214 & 0.4 & 1.7 \\
\hline airplane & 256 & 145,657 & 4.445 & 142,963 & 4.362 & 1.8 & 1.9 & 155,603 & 4.748 & 152,647 & 4.658 & 1.9 & 1.9 \\
\hline anemone & 256 & 211,103 & 4.966 & 204,085 & 4.801 & 1.5 & 3.3 & 241,394 & 5.678 & 233,559 & 5.494 & 1.5 & 3.2 \\
\hline arial & 256 & 280,074 & 6.183 & 280,074 & 6.183 & 1.0 & 0.0 & 294,979 & 6.512 & 293,722 & 6.484 & 0.6 & 0.4 \\
\hline baboon & 256 & 212,881 & 6.496 & 211,123 & 6.442 & 1.5 & 0.8 & 219,469 & 6.697 & 218,264 & 6.660 & 1.5 & 0.5 \\
\hline bike3 & 256 & 372,720 & 4.154 & 372,720 & 4.154 & 1.0 & 0.0 & 434,896 & 4.847 & 434,896 & 4.847 & 1.0 & 0.0 \\
\hline boat & 256 & 190,834 & 5.823 & 184,279 & 5.623 & 2.0 & 3.4 & 199,142 & 6.077 & 191,779 & 5.852 & 2.0 & 3.7 \\
\hline clegg & 256 & 488,553 & 5.456 & 482,503 & 5.388 & 1.3 & 1.2 & 548,572 & 6.126 & 543,946 & 6.074 & 1.3 & 0.8 \\
\hline cwheel & 256 & 172,718 & 2.878 & 171,273 & 2.854 & 0.8 & 0.8 & 194,616 & 3.243 & 192,291 & 3.204 & 0.8 & 1.2 \\
\hline fractal & 256 & 282,417 & 5.828 & 273,932 & 5.653 & 1.6 & 3.0 & 292,557 & 6.038 & 283,206 & 5.845 & 2.2 & 3.2 \\
\hline frymire & 256 & 521,446 & 3.376 & 514,140 & 3.329 & 0.5 & 1.4 & 651,322 & 4.217 & 629,749 & 4.078 & 0.6 & 3.3 \\
\hline ghouse & 256 & 272,465 & 4.541 & 269,666 & 4.494 & 1.3 & 1.0 & 305,487 & 5.091 & 302,681 & 5.044 & 1.3 & 0.9 \\
\hline girl & 256 & 172,202 & 5.255 & 170,288 & 5.196 & 1.1 & 1.1 & 181,728 & 5.545 & 180,209 & 5.499 & 1.2 & 0.8 \\
\hline house & 256 & 39,767 & 4.854 & 39,447 & 4.815 & 1.4 & 0.8 & 42,106 & 5.139 & 41,791 & 5.101 & 1.4 & 0.8 \\
\hline lena & 256 & 165,457 & 5.049 & 159,620 & 4.871 & 1.9 & 3.5 & 178,097 & 5.435 & 168,597 & 5.145 & 1.9 & 5.3 \\
\hline monarch & 256 & 192,548 & 3.917 & 192,548 & 3.917 & 1.0 & 0.0 & 222,169 & 4.520 & 222,169 & 4.520 & 1.0 & 0.0 \\
\hline peppers & 256 & 164,481 & 5.019 & 155,068 & 4.732 & 1.6 & 5.7 & 176,898 & 5.398 & 166,909 & 5.093 & 1.7 & 5.6 \\
\hline serrano & 256 & 204,369 & 3.273 & 198,111 & 3.173 & 0.7 & 3.1 & 269,893 & 4.323 & 260,911 & 4.179 & 0.6 & 3.3 \\
\hline tulips & 256 & 198,226 & 4.032 & 198,226 & 4.032 & 1.0 & 0.0 & 228,854 & 4.656 & 228,755 & 4.654 & 1.8 & 0.0 \\
\hline Average & - & - & 3.122 & - & 3.077 & - & 1.4 & - & 3.521 & - & 3,460 & - & 1.7 \\
\hline
\end{tabular}

Table 1. Lossless compression results, using JPEG-LS and lossless JPEG 2000 encoders, of a number of synthetic and natural color-indexed images. The "mZeng" values refer to the technique proposed in [17], whereas "Proposed" refers to the reordering method based on the exponential power distribution model addressed in this paper. "Gain" indicates the percentage of compression of the "Proposed" in relation to the corresponding "mZeng". The $\gamma$ columns indicate the best value of this parameter for each image / encoder pair. All compression values include the size of the color-map.

Proc. of the 7th IEEE Int. Conf. on Image Processing, ICIP-2000, vol. III, Vancouver, Canada, Sept. 2000, pp. 476-479.

[15] A. Spira and D. Malah, "Improved lossless compression of color-mapped images by an approximate solution of the traveling salesman problem," in Proc. of the IEEE Int. Conf. on Acoustics, Speech, and Signal Processing, ICASSP-2001, vol. III, Salt Lake City, UT, May 2001, pp. 1797-1800.
[16] S. Battiato, G. Gallo, G. Impoco, and F. Stanco, "A color reindexing algorithm for lossless compression of digital images," in Proc. of the IEEE Spring Conf. on Computer Graphics, Budmerice, Slovakia, Apr. 2001, pp. 104-108.

[17] A. J. Pinho and A. J. R. Neves, "A note on Zeng's technique for color reindexing of palette-based images," IEEE Signal Processing Letters, vol. 11, no. 2, Feb. 2004. 